This article has been scanned by iThenticat No plagiarism detected

Volume 3, Issue 6, December 2021

p. $451-457$

\title{
SODIUM CHLORIDE (SALT) ITS ROLE AND USE IN ANCIENT IRAQ
}

http://dx.doi.org/10.47832/2757-5403.6-3.33

Shaymaa Issam Alawi ALBALDAWI ${ }^{1}$ \& Faten Hameed Qasim AL SARRAJI²

\begin{abstract}
:
Mineral Salts are One of the Most important Non-Metallic Chemicals that were widely available in ancient Iraq, and due to its many Uses in life Matters and for industrial purposes, it was Allocated in the Study of a Type of these Salts, which is Sodium Chloride salt (Table Salt), as there were Several Types, including: - Calcium Sulfate Sodium Silicate, Sodium Carbonate and Potassium Nitrate Because of the Importance of Sodium Chloride (salt) in Ancient Iraq and the lack of Studies that Dealt with it, we Chose it as a Subject for our Study, and we Relied on Many Arab, Translated and Foreign Sources, the most important of which are: -Levy, Martin, 1980 , Chemistry and Chemical Technology, translated by: Al-Mayahi and others, Dar Al-Rashid.

- Potts ,D., 1987," ON Salt and salting gathering in Ancient Mesopotamia "(JESHO), vol.27.
\end{abstract}

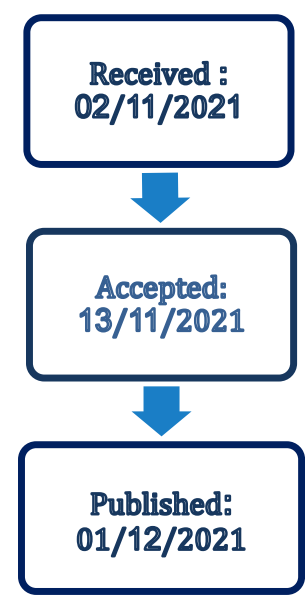

Key words: Sodium Chloride, Salt, Gold, Diseases, Meat preservation.

\footnotetext{
${ }^{1}$ Researcher, Kufa University, Iraq

${ }^{2}$ Dr. , Imam Ja'afar Al-Sadiq University, Iraq, fatin.hameed@sadiq.edu.iq, https://orcid.org/0000-0003-2300$\underline{3696}$
}

Copyright (C) Published by IJHER Journal, www.ijherjournal.com Rimar Academy, Fatih, Istanbul, 34093 Turkey

All rights reserved 
مادة كلوريد الصوديوم (الملح) دورها واستخدامها في العراق القديم

3

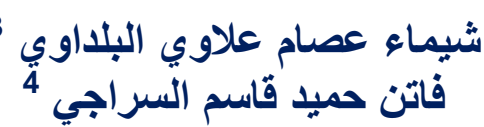

الملخص

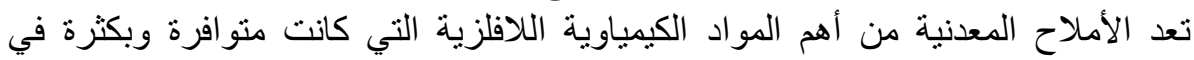

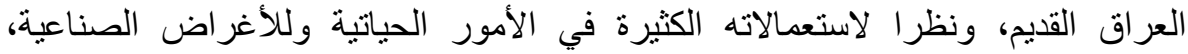

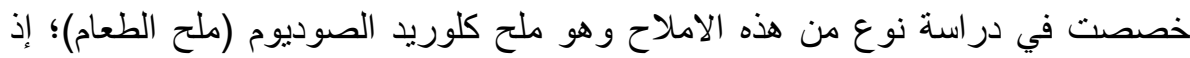

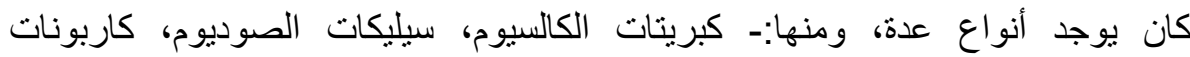

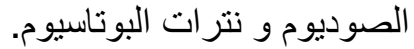
و لأهمية مادة كلوريد الصوديوم (الملح) في العراق القديم ولقلة الدراسات التي تنتاولته

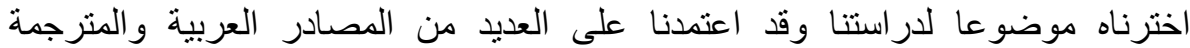

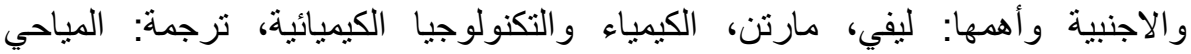
و آخرون، دار الرشيد، و الأنها:

- Potts ,D.,1987," ON Salt and salting gathering in Ancient

Mesopotamia "(JESHO), vol.27 الكلمات المفتاحية: كلوريد الصوديوم، الملح، الذهب، الأمر اض، حفظ اللحوم.

((تعرف السماء أنّ الحياة المتحضرة مستحيلة بلا ملح))، هي عبارة قديمة ترجع إلى زمن الكاتب الكانب والرحالة الإغريقي

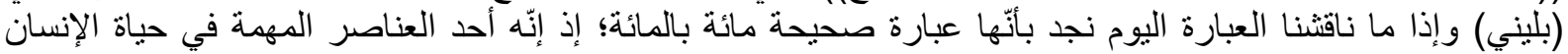

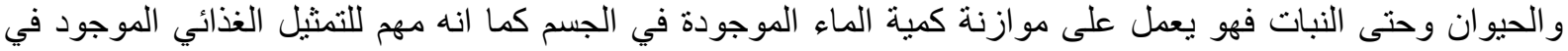

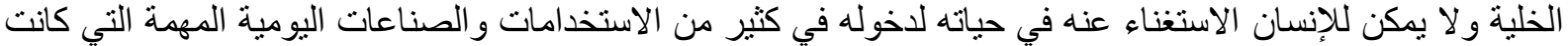

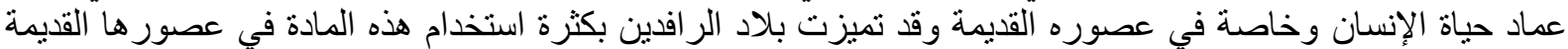

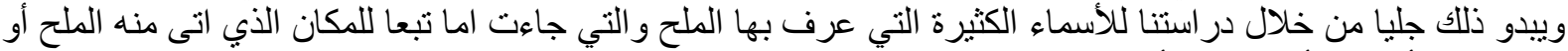

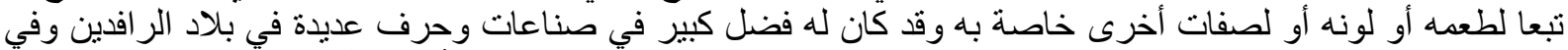

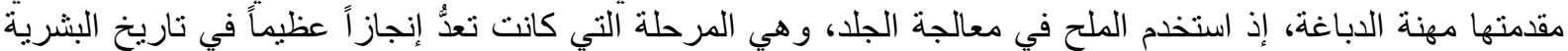

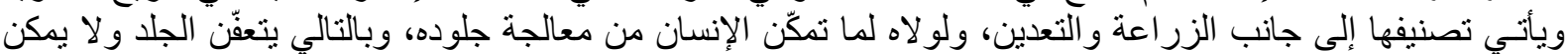

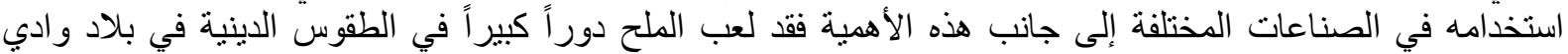

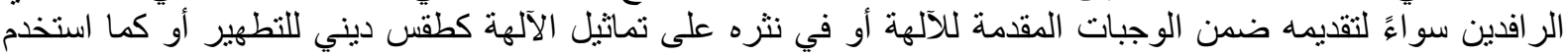

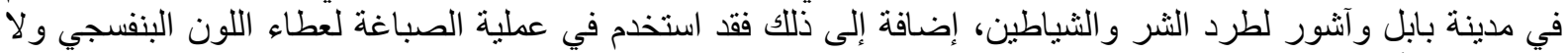

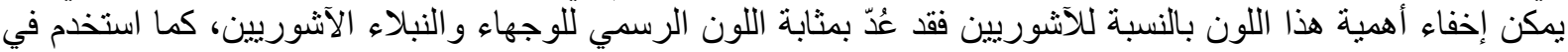

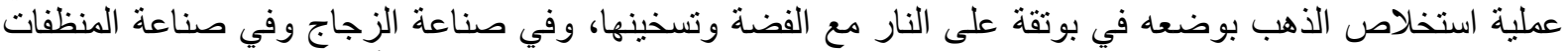

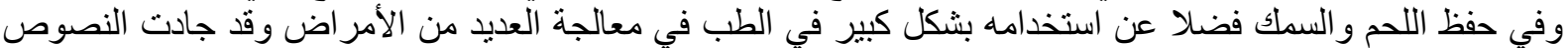

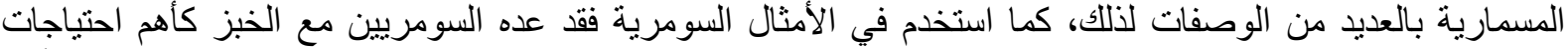

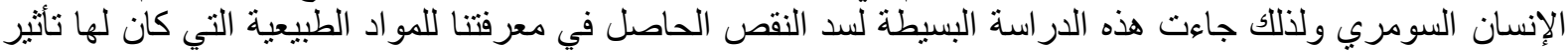

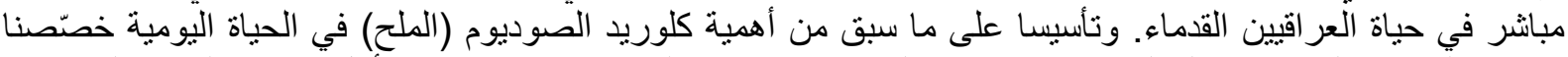

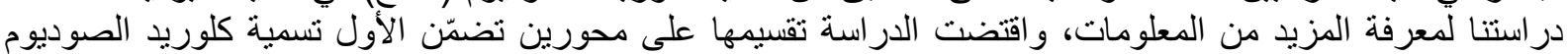
(الملح) وتركيبه الكيمياوي ومصني المعادره، أمّا المحور الآخر فكان حول استعمالاته العديدة.

3 الباحثة، جامعة الكوفة، العراق

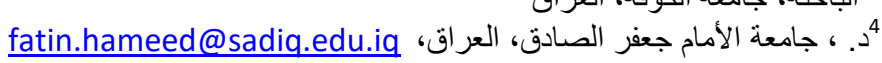




\section{المحور الأول - تسمية الملح - التركيب الكيمياوي- مصادره

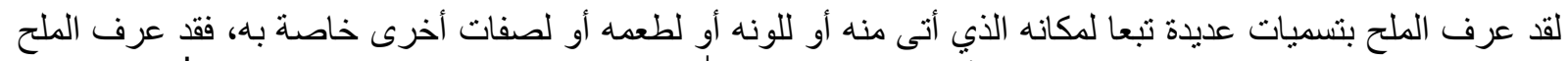

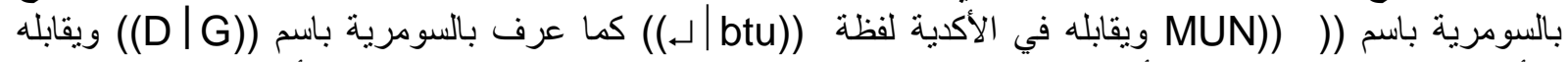

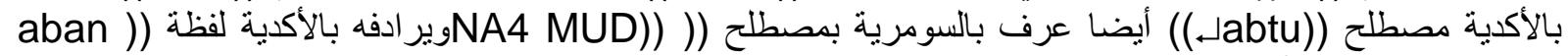

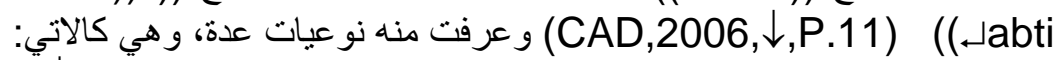

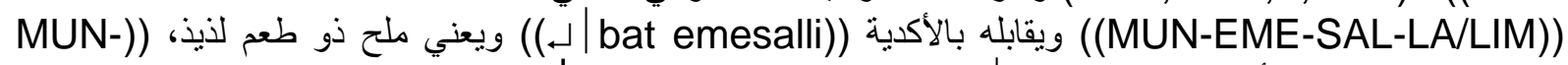

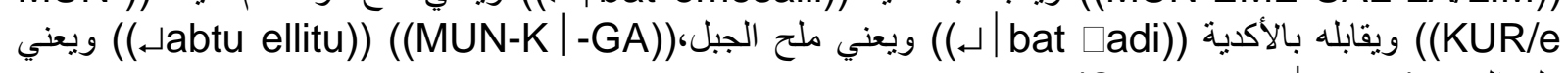

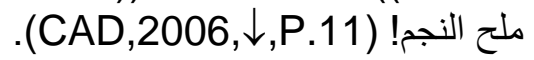

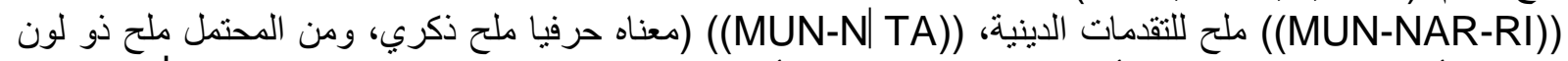

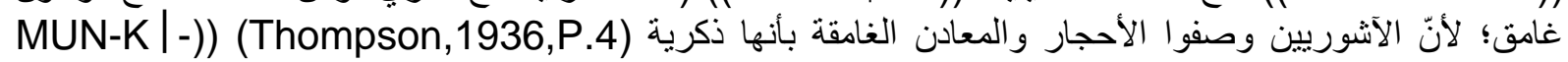
((MIN)) ((MUN-GAG--UB-BA))،((MUN-BAR-SA-TU-RA)) ، (MUN ba-ri)) ((PAD

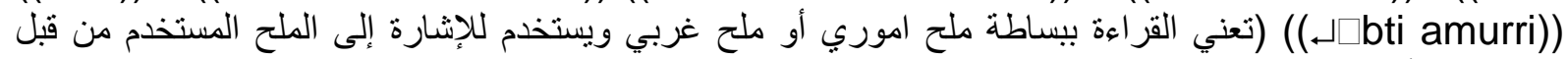

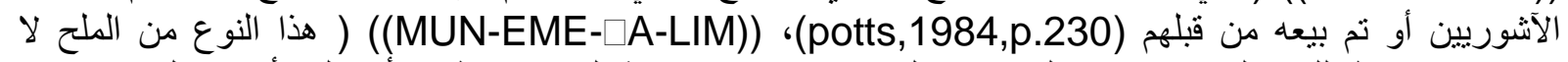

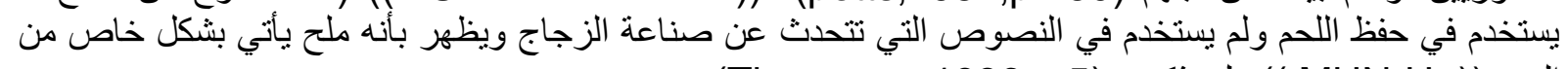

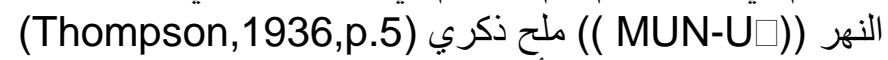

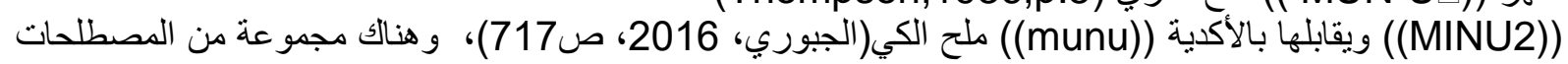

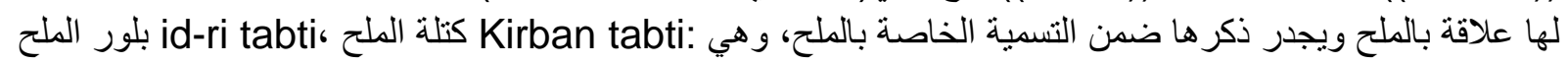

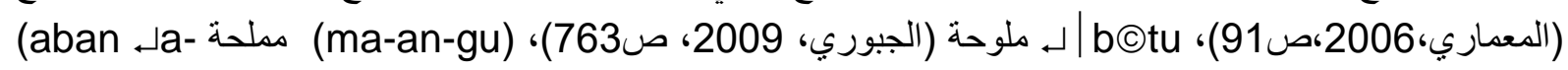
ab-ti NA4 MUN)

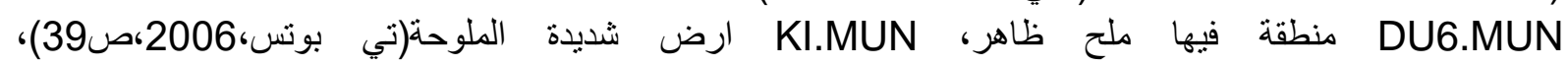

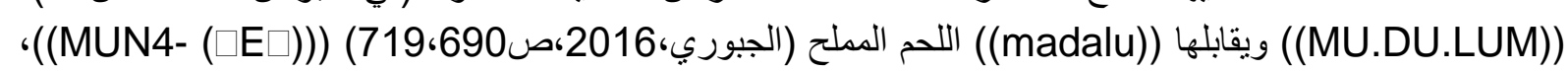

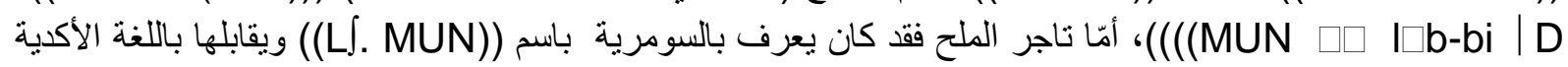

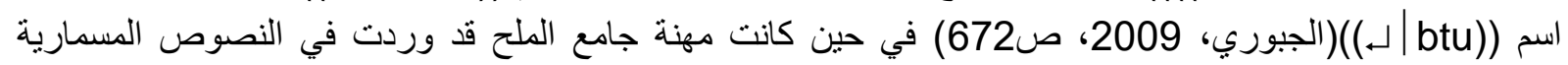

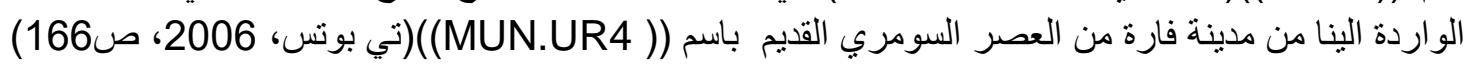

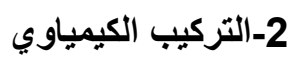

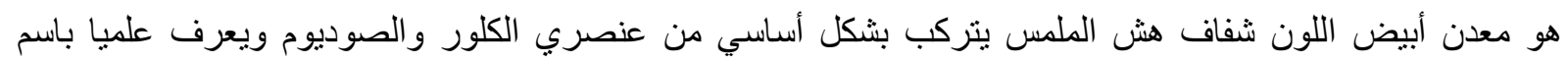

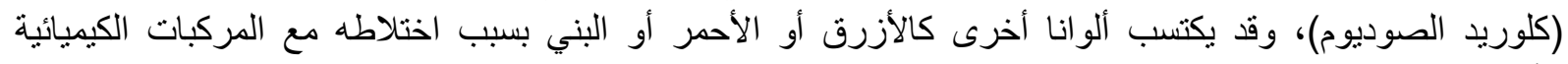

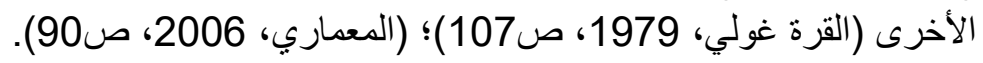

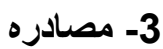

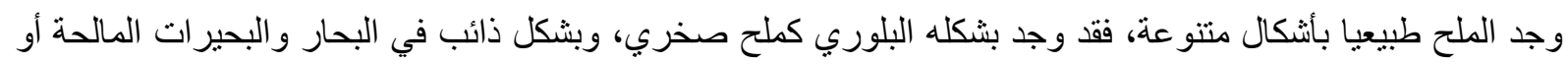

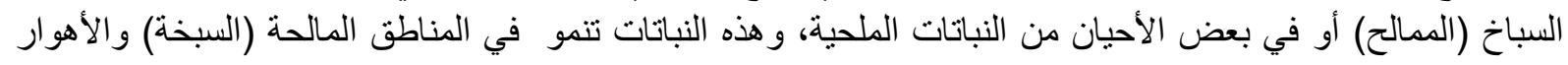

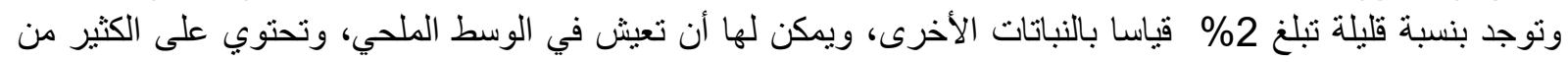

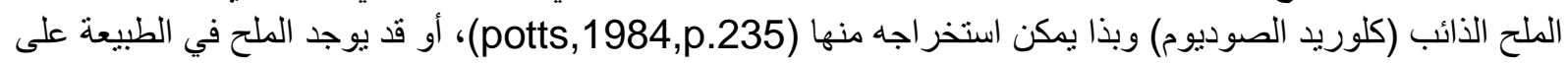

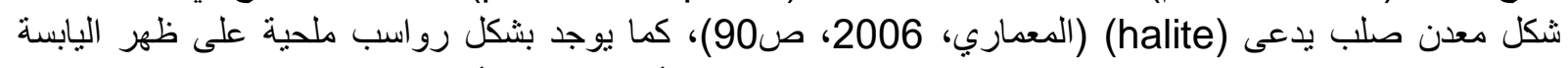
وتختلف هذه الطقات في سمكها ويوجد في بعض (البلاد على سطح الأرض اليابسة أو تحت سطحها في الدناجم (عباش، 1967، صن صن (20) 
ان واحدة من اهم ممالح العراق القديم هي مملحة السماوة وهي قريبة من اور و الوركاء، كما تذكر النصوص الأدبية في

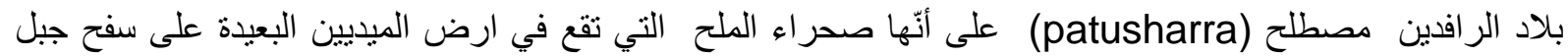

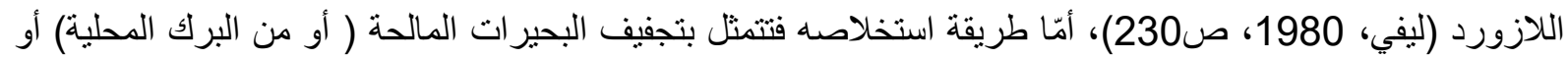

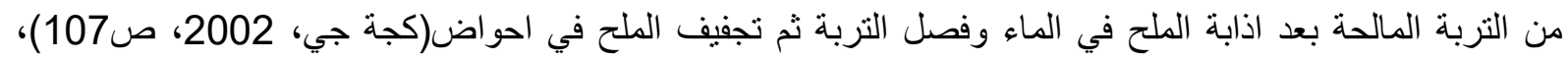

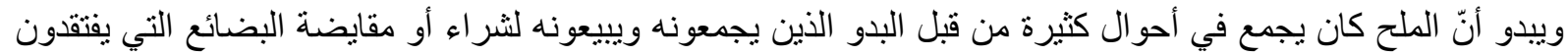

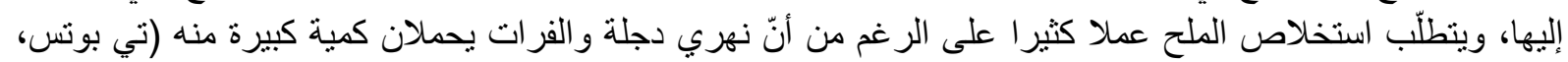

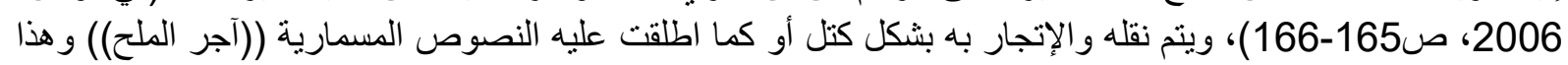

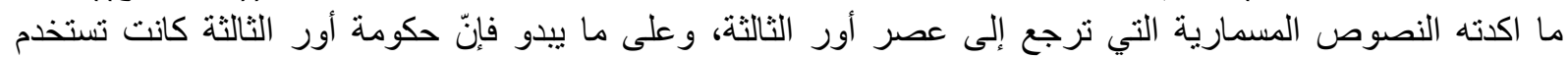

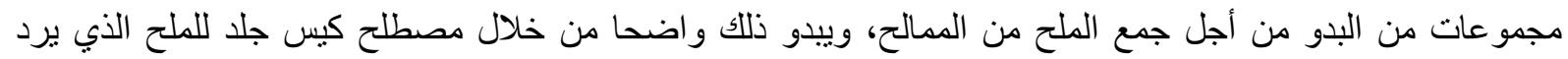

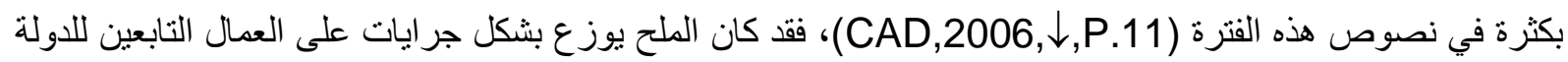

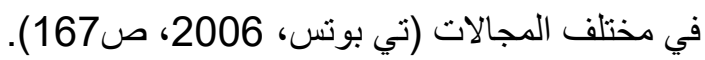

المحور الثاني -استعمالات الملح فالح الماتح

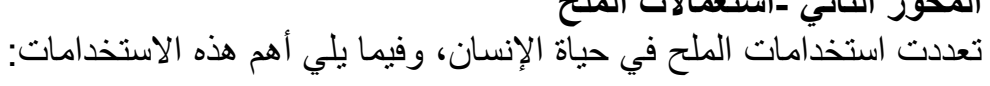

1- 1 الثعائر والطقوس الدينية:

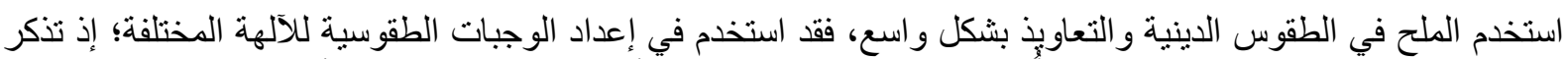

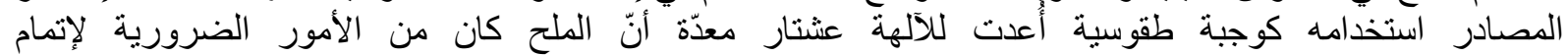

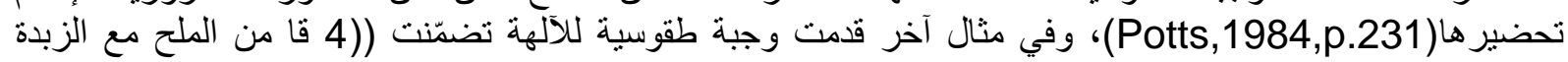

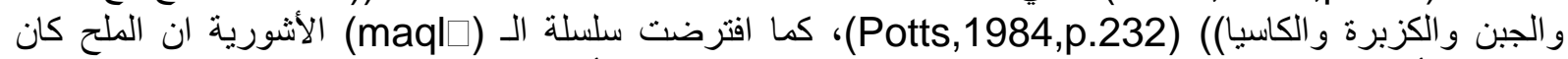
ضروريا لأعداد الوجبات الطقوسية للآلهة، ومر الفقة لحرق البخور حيث نقرأ في تعويذة لتمجيد الملح:

يا ملح، خلقت في مكان نظيف

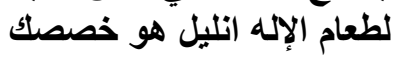

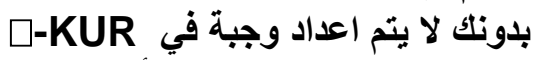

باونك الإله، الملك، السيد، الأمير لا يشم رائحة البخور(Potts,1984,pp.231-232).

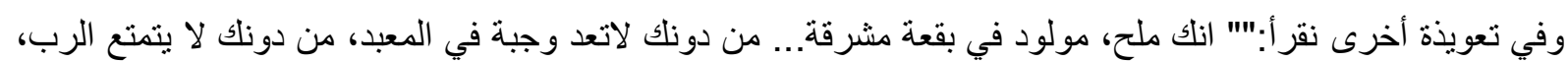

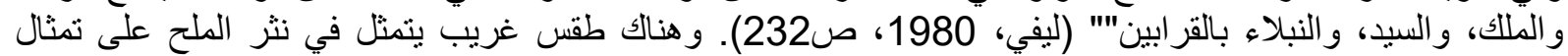

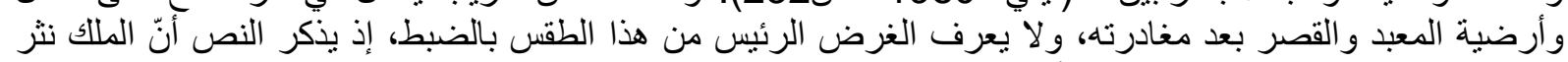

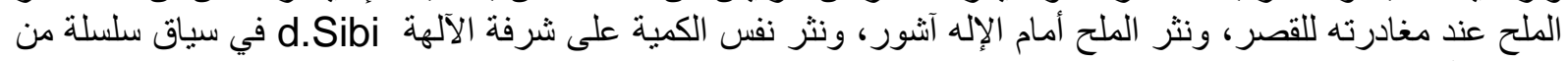

طقوس التقديم (Potts, 1984,p.232)

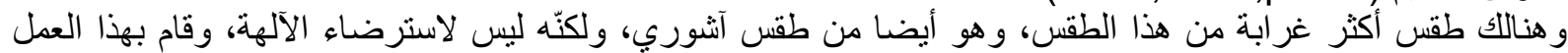

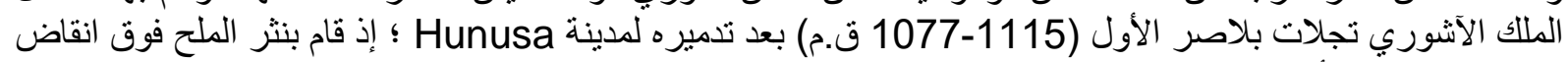

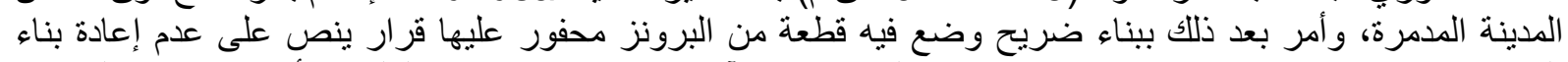

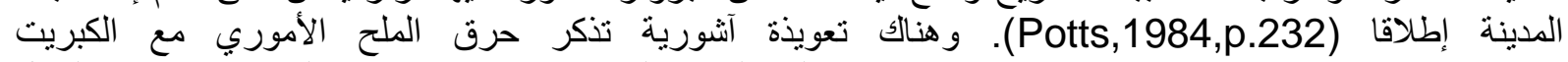
(Meier,1937,p.79) والماء)(Potts, 1984,p.230).

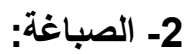

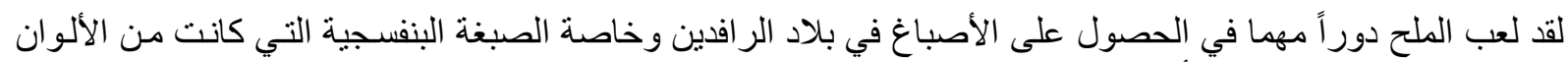

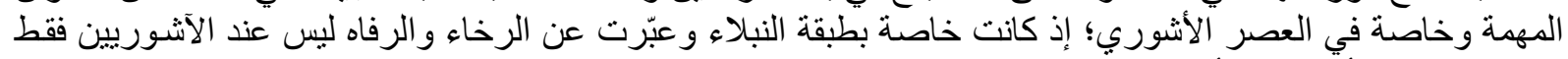

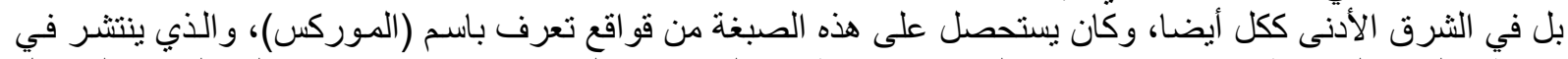

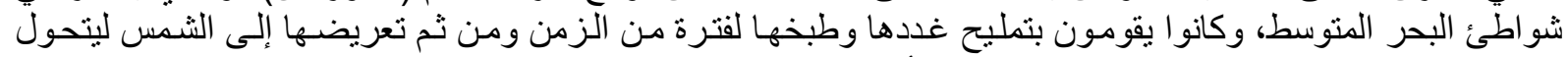

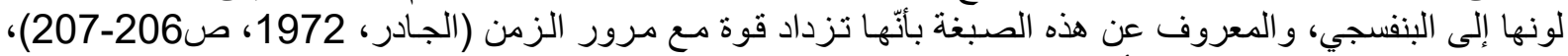

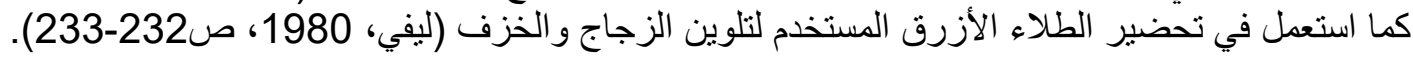




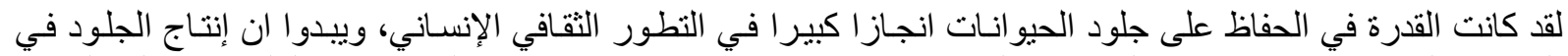

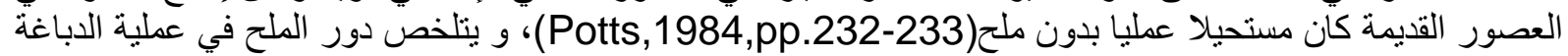

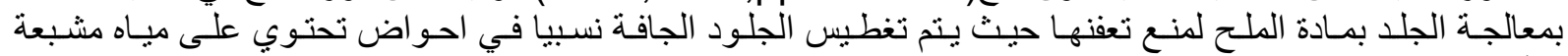

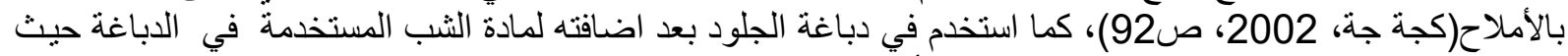

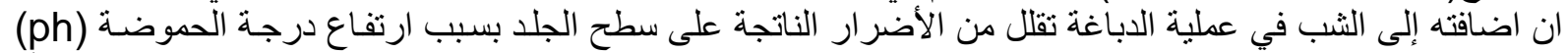

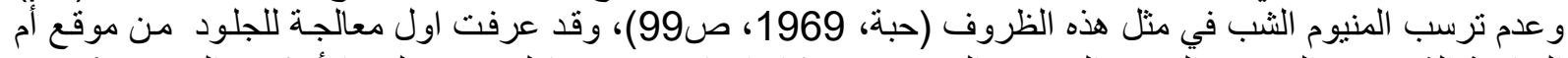

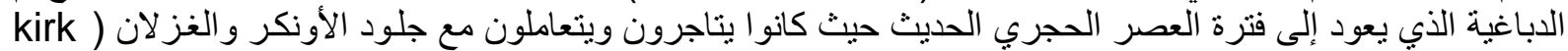

.(bride,1974,pp.85-92

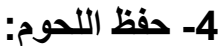

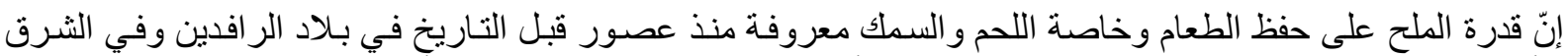

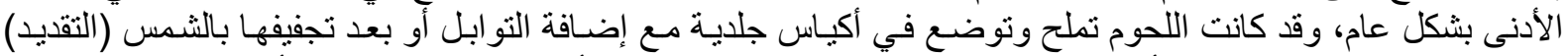

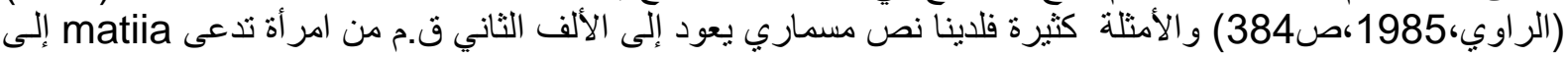

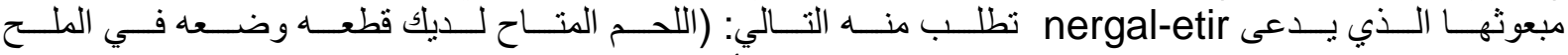

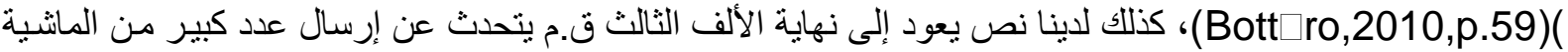

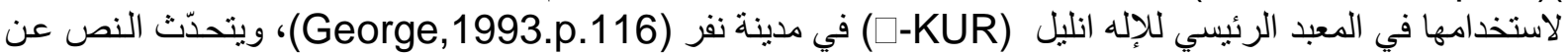

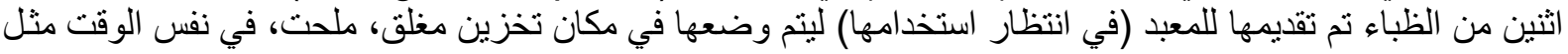

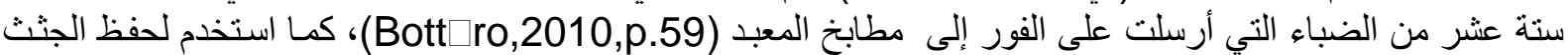

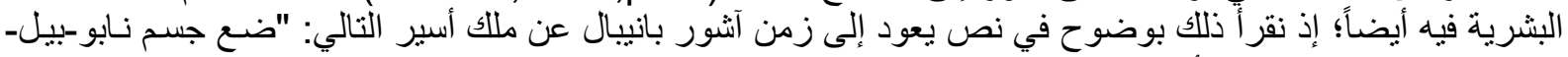

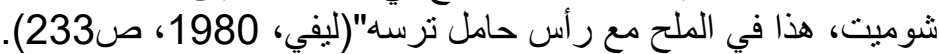
5- 5 استخلاص الذهب وتنقيته :

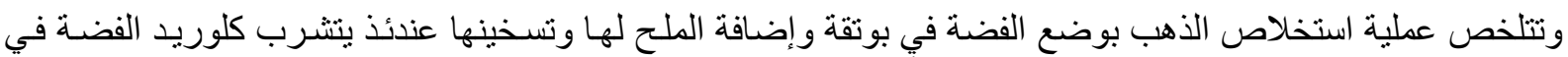

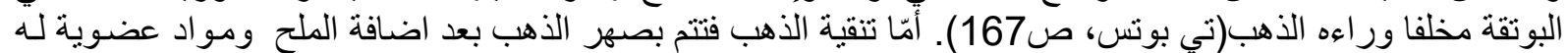

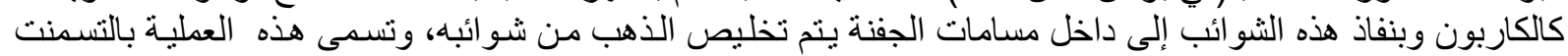

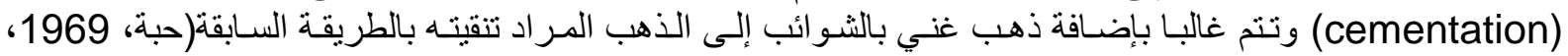

ص167). (167)

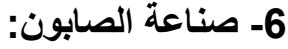

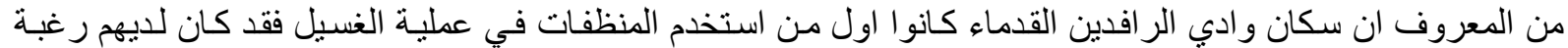

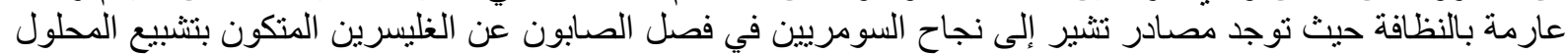

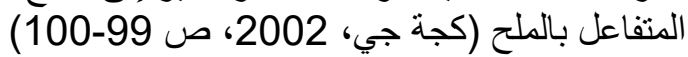

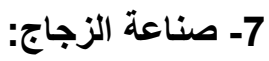

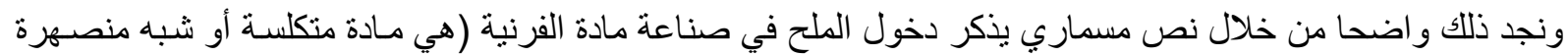

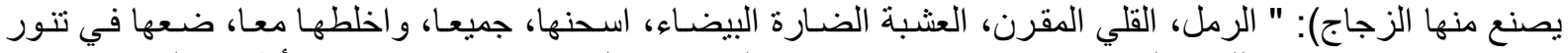

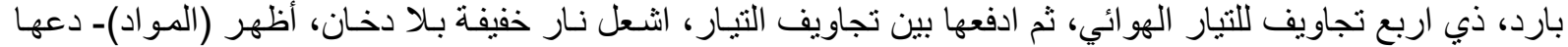

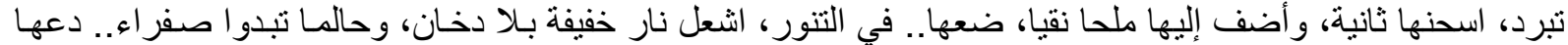

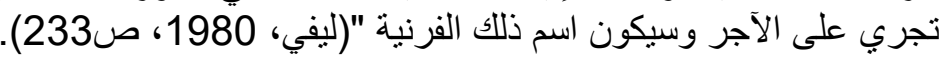

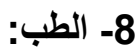

استعملت مادة الملح في الطب بكثرة إذ كان يؤخذ للاستعمال الداخلي و الخارجي لمعالجة مختلف الأمر اض ومنها: 


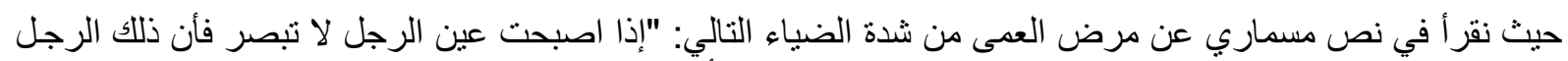

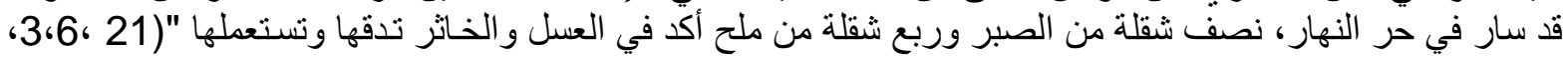

(Thompson ,1923

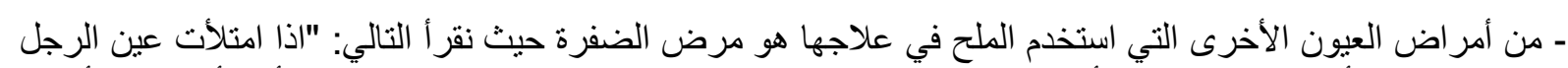

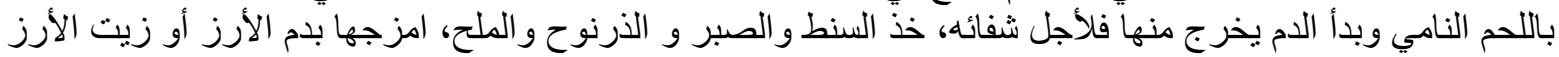

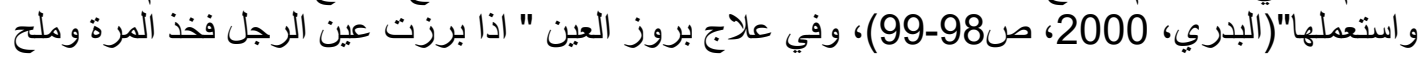

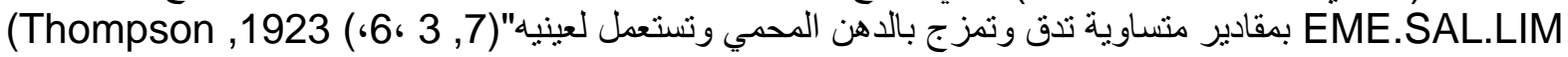
ب- أمراض الأذن: كان الملح يوضع على قطعة من الصوف مع زيت شجر الأرز ويدخل في الأذن(ليفي، 1980، ص233).

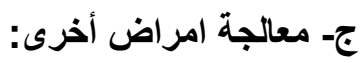

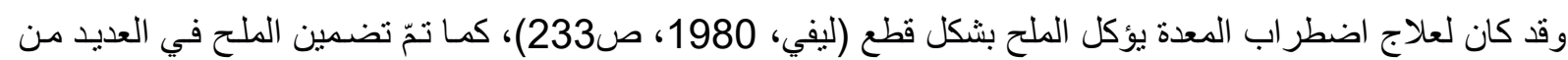

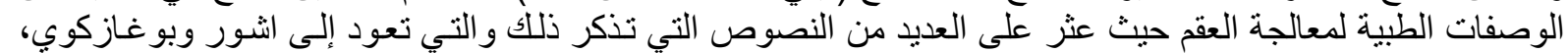
ايضا استخدم الملح كمخدر واستخدم بشكل لا يقل أهمية في علاج الأمر أض النفية النفية (Potts, 1984,1984,p.230)

9- 9 استخد/مه على المائدة:

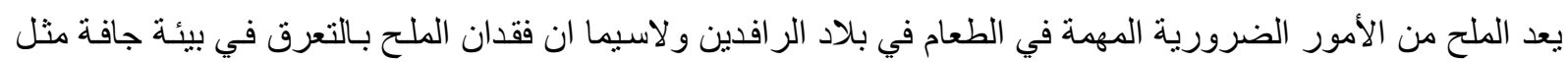

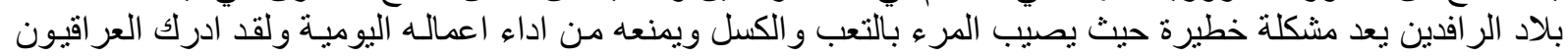

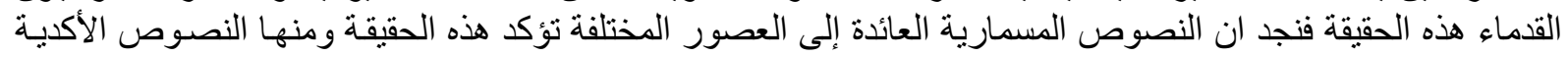

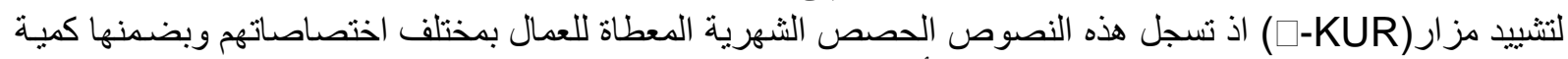

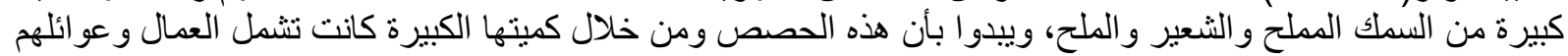

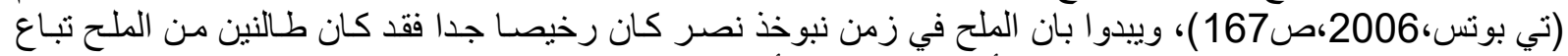

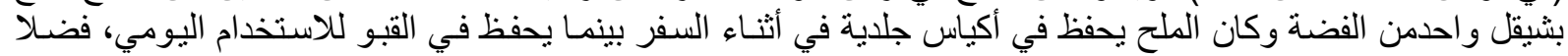

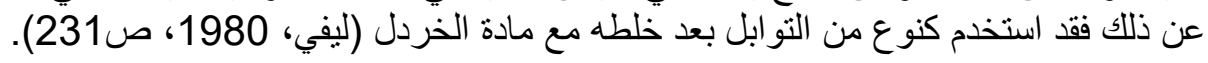

\section{0- استعمالاته في أشياء أخرى:}

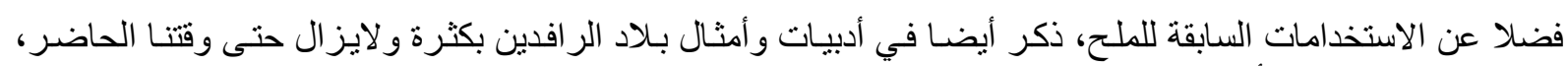

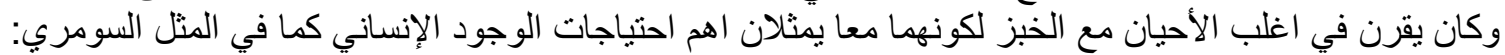

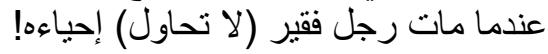

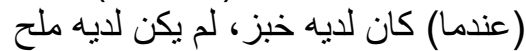

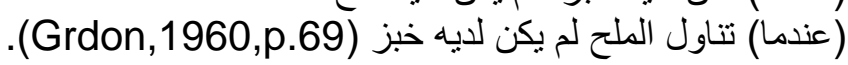

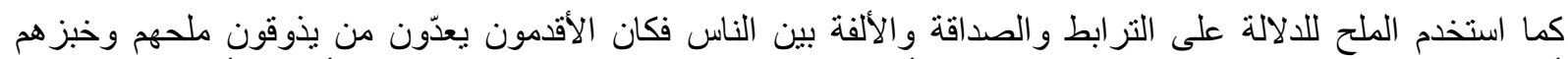

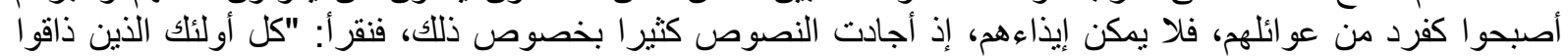

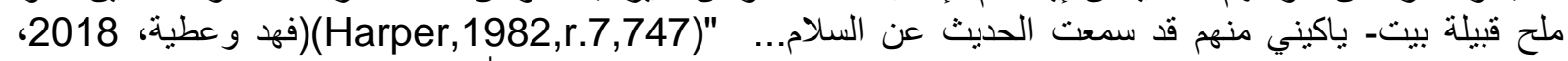
صاب268)، دخل في تركيب أسماء العائلات أيضا في العر اق القديم ومنها: (bti

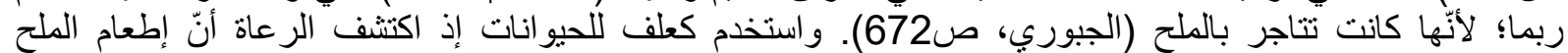

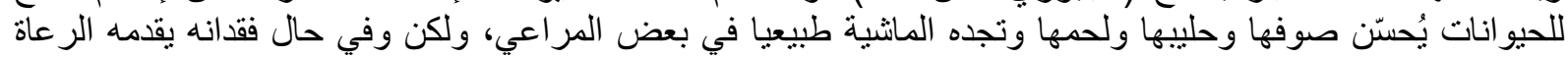

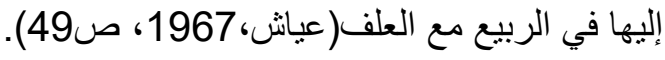

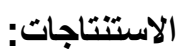

من خلال ما تقدّم من هذا البحث يمكننا أنْ نستنتج مجمو عة من الاستنتاجات المهمة، وهي كالتالي:

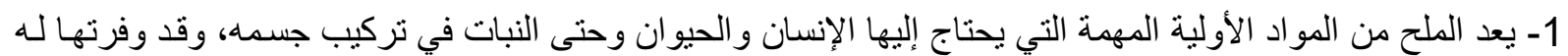

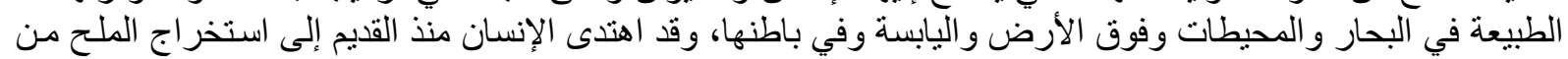

مصنادره الطبيعية. 


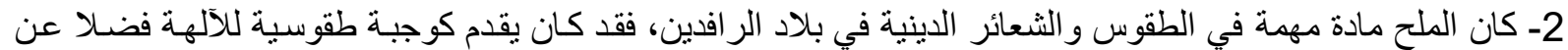

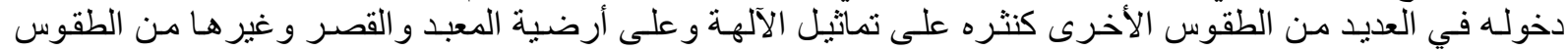
المختلفة

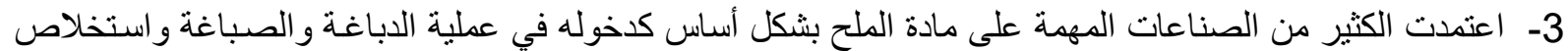

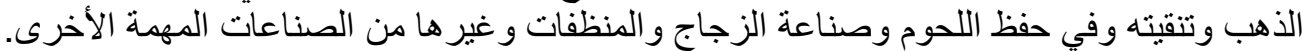

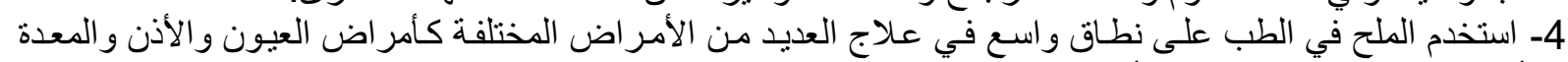

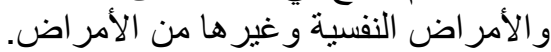

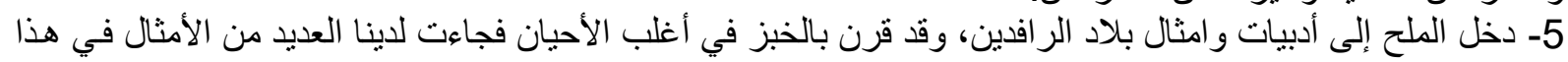

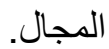

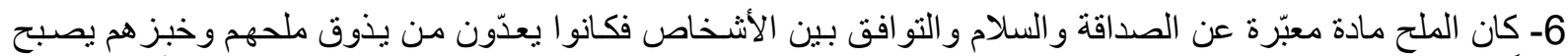

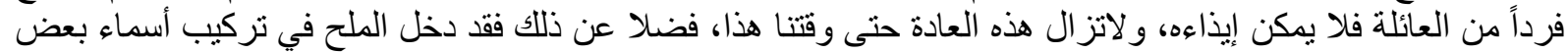

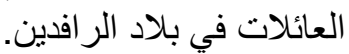

أولا: المصادر العربية

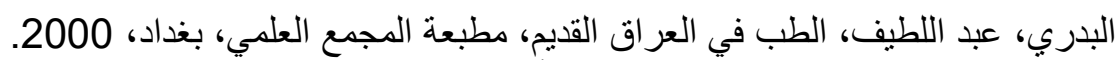

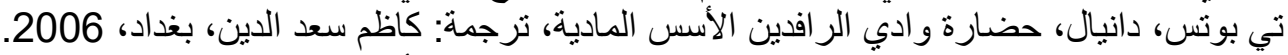

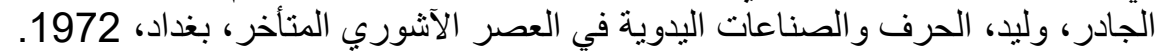

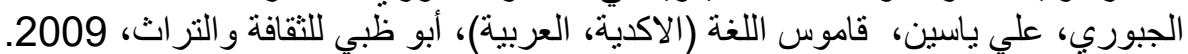

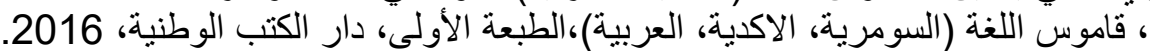

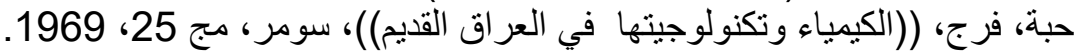

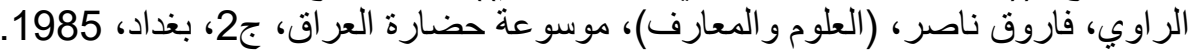

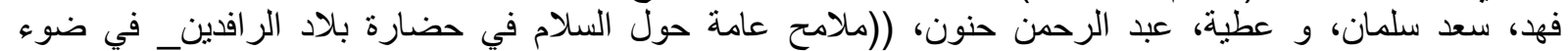

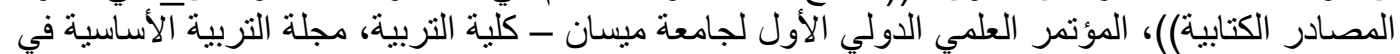

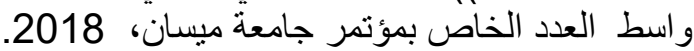

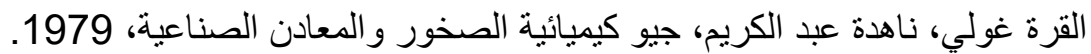
عباش، عبد القادر، الملح في حياتتا وتراثنا، سوريا،

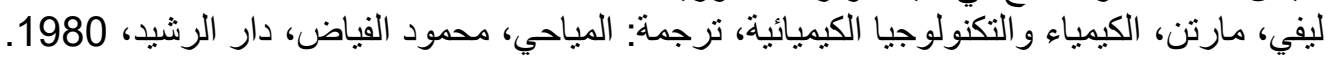

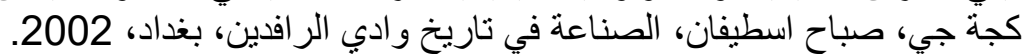

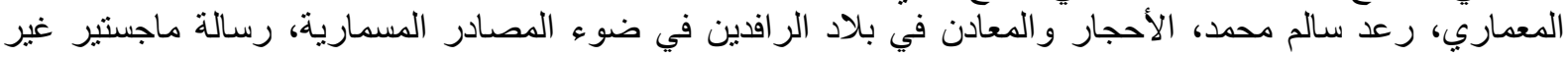
منشورة، جامعة الموصل، 2006.

\section{ثانياً: المصادر الأجنبية}

Black, J. and others,2000, A Concise Dictionary of Akkadian, Wiesbaden.

Borger , R., 1987, Assyrisch- Babylonisch Zeichenliste, Germany.

Botter ,J.,2010, Ancient Mesopotamia Cuisin, Paris.

George, A.R., 1993, House Most High the Temples of Ancient Mesopotamia, Eisenbrauns.

Grdon,E.I.,1960, Sumerian Proverbs 'Glimpses of everyday Life in Ancient Mesopotamia, University Museum, Philadelphia.

Harper, R.F.,1982, Assyrian and Babylonian Letters, Chicago, Vol.1.

Kirk bride, D.,1937," Umm Dabaghiyab: a Trading out post ", Iraq ,Vol.36

Labat, R., 1967, Manual D'epigraphie Akkadienne, MDA, Paris.

Meier,G., 1937,"Die A $\square \square$ yrische Beschw $\square$ rungsirtule Maql $\square "$, AFO, Beiheft2,Berlin.

Oppenheim, A., L., and others,2006, The Assyrian Dictionary of the Oriental Institute of the University of Chicago (CAD), Chicago.

Potts ,D.,1987," ON Salt and salting gathering in ancient Mesopotamia "(JESHO), vol.27.

Schramm, W., 2003, Akkadisch Logogramme, GAAL4, Gottingen.

Thompson, R.C.,1923, Assyrian Medical Texts from the Originals in the British Museum (AMI), Oxford.

1936, Dictionary of Assyrian Chemistry and Geology, (DACG) Oxford. 